\title{
Fusion of Magnetic Resonance Angiography and Magnetic Resonance Imaging for Surgical Planning for Meningioma
}

\author{
-Technical Note-
}

\author{
Hiroshi Kashimura, Kuniaki Ogasawara, Hiroshi Arai, Takaaki BePpU, \\ Takashi INOUE*, Tsutomu TAKAHASHI**, Koichi MATSUDA**, \\ Yujiro TAKAHASHI**, Shunrou FUJIWARA***, and Akira OGAWA \\ Department of Neurosurgery, Iwate Medical University School of Medicine, \\ Morioka, Iwate; * Department of Neurosurgery, Kohnan Hospital, Sendai, Miyagi; \\ **Software and Information Science, Iwate Prefectural University, Iwate; \\ ***Advanced Medical Research Center, Takizawa, Iwate
}

\begin{abstract}
A fusion technique for magnetic resonance (MR) angiography and MR imaging was developed to help assess the peritumoral angioarchitecture during surgical planning for meningioma. Three-dimensional time-of-flight (3D-TOF) and 3D-spoiled gradient recalled (SPGR) datasets were obtained from 10 patients with intracranial meningioma, and fused using newly developed volume registration and visualization sof tware. Maximum intensity projection (MIP) images from 3D-TOF MR angiography and axial SPGR MR imaging were displayed at the same time on the monitor. Selecting a vessel on the realtime MIP image indicated the corresponding points on the axial image automatically. Fusion images showed displacement of the anterior cerebral or middle cerebral artery in 7 patients and encasement of the anterior cerebral arteries in 1 patient, with no relationship between the main arterial trunk and tumor in 2 patients. Fusion of MR angiography and MR imaging can clarify relationships between the intracranial vasculature and meningioma, and may be helpful for surgical planning for meningioma.
\end{abstract}

Key words: three-dimensional time-of-flight magnetic resonance angiography, magnetic resonance imaging, meningioma, fusion imaging

\section{Introduction}

Surgical planning for meningioma requires information about the peritumoral angioarchitecture, in addition to the tumor consistency, location, and size, ${ }^{5,12)}$ because the involvement or course of any adjacent arteries affects the surgical outcome in tumors located at the skull base or eloquent areas. ${ }^{6)}$ Magnetic resonance (MR) imaging provides good spatial resolution and excellent contrast with soft tissues, but does not allow detailed analysis of the cerebral vasculature. MR angiography provides just such information on cerebral vasculature. ${ }^{2)}$ Therefore, fusing of the MR angiograms and MR images may enable simultaneous visualization of the intracranial vasculature and brain tissues.

The present study developed a fusion technique

Received November 19, 2007; Accepted April 22, 2008 for MR angiography and MR imaging that could facilitate surgical planning for meningioma.

\section{Technical Description}

MR imaging was performed with a Signa VH/i 3.0 T scanner (General Electric Systems, Milwaukee, Wis., U.S.A.) and a parallel imaging head coil. Three-dimensional time-of-flight (3D-TOF) MR angiography was performed using conventional singleslab acquisition in all patients. The pulse sequence was as follows: repetition time (TR), $30 \mathrm{msec}$; echo time (TE), $3.9 \mathrm{msec}$; flip angle, $60^{\circ}$; matrix size, 512 $\times 256$; field of view (FOV), $22 \times 17 \mathrm{~cm}$; slice thickness, $1.2 \mathrm{~mm}$; 1 averaged with an acquisition time of 11 minutes 12 seconds. Then, 3D-spoiled gradient recalled (SPGR) MR imaging was acquired using the following: TR, $11.3 \mathrm{msec}$; TE, $2.2 \mathrm{msec}$; flip angle, $15^{\circ}$; matrix size, $512 \times 256$; FOV, $22 \times 22 \mathrm{~cm}$; slice thickness, $1.2 \mathrm{~mm}$; 1 averaged with an acquisition 
time of 5 minutes 35 seconds.

The 3D-TOF and 3D-SPGR datasets were fused on a personal computer using volume registration and visualization software that we developed. This original software was implemented with $\mathrm{C} / \mathrm{C}++$ on Windows by one of the authors (T.T.). The software supports the following formats: DICOM and ANALYZE 7.5. Coordination between the two volume datasets was manually adjusted if required, although 3D-TOF and 3D-SPGR were performed sequentially. The registration procedure started with manual designation of a predefined anatomic point in both modalities. This procedure requires rigid-motion transformation (rotation, translation, scaling) between 3DMR imaging and 3D-MR angiography. Scaling can be derived from knowledge of the voxel sizes in both modalities, so only rotation and translation had to be estimated from the data. Therefore, new volume registration software was developed to rotate and translate one volume dataset through a graphical user interface to visualize two volume datasets in different colors within the same 3D space using realtime volume rendering. All volume registration was successfully performed within 15 minutes per patient.

Using this software, the maximum intensity projection (MIP) of 3D-TOF MR angiography and axial SPGR MR imaging were displayed simultaneously on the monitor. Selecting the vessel on the real-time MIP image with the mouse resulted in software indicating the corresponding points on the axial image automatically. Using this method, we could evaluate the accuracy of registration between vessels in 3D-MR angiography and hyperintense areas on axial MR imaging, which corresponded to the vessels on SPGR MR images.

The software was based on a new algorithm for visualizing vessel tracts. A green line was traced along the vessels on the MIP images in 3D space and the green line was shown simultaneously on the axial SPGR MR image as a red point. In addition, the positions of vessels could also be visualized on 3DMR angiography by selecting the corresponding vessels on the two-dimensional (2D) image. Therefore, the correspondence between any artery on 3D-MR angiography and a hyperintense spot or string on axial MR imaging could easily be demonstrated in real time.

\section{Results}

MR fusion imaging was successfully performed within 15 minutes in a total of 10 patients, 7 women and 3 men aged 29-73 years (mean 54.9 years), with meningioma between December 2006 and April 2007 (Table 1). The tumor was located in the convex in 4 patients, the falx in 1 , the cerebellar tentorium in 1 , the petroclivus in 1 , the temporal base in 1 , the olfactory groove in 1 , and the tuberculum sellae in 1. All tumors were surgically resected

Table 1 Summary of 10 patients

\begin{tabular}{|c|c|c|c|c|c|c|c|}
\hline $\begin{array}{l}\text { Case } \\
\text { No. }\end{array}$ & $\begin{array}{c}\text { Age } \\
\text { (yrs)/ } \\
\text { Sex }\end{array}$ & $\begin{array}{l}\text { Tumor } \\
\text { location }\end{array}$ & $\begin{array}{l}\text { Tumor } \\
\text { size } \\
(\mathrm{cm})\end{array}$ & \multicolumn{3}{|c|}{ Peritumoral angioarchitecture } & $\begin{array}{c}\text { Postoperative } \\
\text { new } \\
\text { neurological } \\
\text { deficits }\end{array}$ \\
\hline 1 & $48 / \mathrm{F}$ & $\begin{array}{r}\text { lt cerebellar } \\
\text { tentorium }\end{array}$ & $5.4 \times 5$ & $\begin{array}{l}\text { BA, lt SCA, } \\
\text { lt PCA }\end{array}$ & & & no \\
\hline 2 & $73 / \mathrm{F}$ & rt parietal falx & $4 \times 3.5$ & & & $\begin{array}{l}\text { rt ACA } \\
\text { branches }\end{array}$ & no \\
\hline 3 & $29 / F$ & rt convex & $7 \times 7$ & & bil $A_{2}-A_{3}$ & & no \\
\hline 4 & $69 / \mathrm{F}$ & lt petroclivus & $3 \times 3$ & BA, lt SCA & & & $\begin{array}{c}\text { lower CN } \\
\text { palsy }\end{array}$ \\
\hline 5 & $38 / \mathrm{M}$ & lt parietal convex & $8.8 \times 6$ & & $\begin{array}{l}\text { lt ACA branch, } \\
\text { lt MCA branch }\end{array}$ & & no \\
\hline 6 & $71 / \mathrm{M}$ & rt frontal convex & $8.8 \times 6$ & & rt $A_{2}-A_{3}$ & & no \\
\hline
\end{tabular}

ACA: anterior cerebral artery, BA: basilar artery, CN: cranial nerve, ICA: internal carotid artery, MCA: middle cerebral artery, PCA: posterior cerebral artery, SCA: superior cerebellar artery. 
and histological diagnoses were established. Appropriate informed consent was obtained from each patient or their relatives.

Fusion images showed displacement of the anterior cerebral artery (ACA) or middle cerebral artery in 7 patients and encasement of the ACA in 1 patient (Table 1). No relationship was seen between the main arterial trunk and the tumor in the remaining 2 patients. The relationship between tumor and arteries corresponded with intraoperative findings in all cases. Only 1 of the 10 patients experienced new neurological deficits, as Case 4 developed lower cranial nerve palsy. However, the symptoms gradually improved. Postoperative MR imaging showed no new ischemic lesions in any patient.
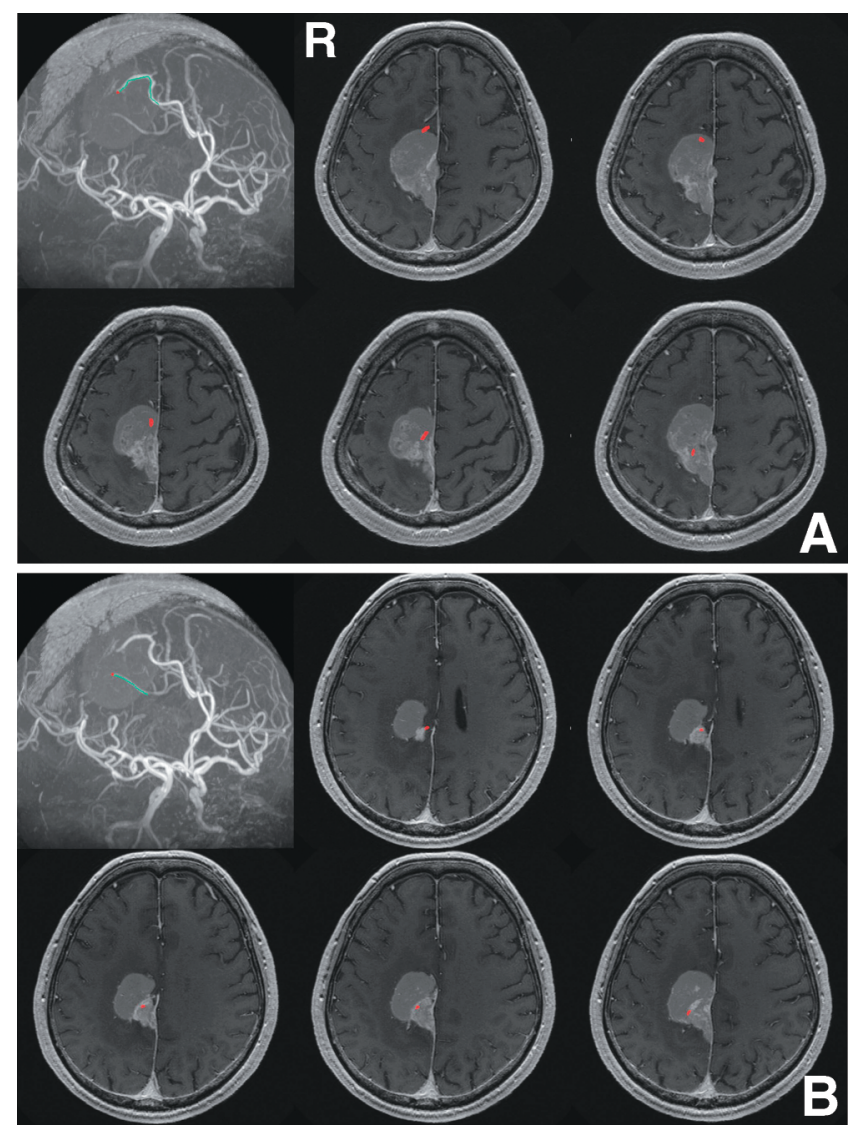

Fig. 1 Case 2, a 73-year-old woman with atypical meningioma. The green line was drawn on the anterior cerebral artery (ACA) on the maximum intensity projection image in three-dimensional space and the location of the green line was shown simultaneously on axial spoiled gradient recalled magnetic resonance images as a red point. The distal branches of the right ACA entered the tumor anteriorly (A) and inferiorly (B), but did not penetrate the tumor.

\section{Illustrative Cases}

Case 2: A 73-year-old woman presented with progressive left leg monoparesis over a period of 1 year. MR imaging revealed heterogeneous enhancement of the tumor attached to the falx in the right cerebral hemisphere. MR fusion imaging clearly showed that the distal branches of the right ACA entered the tumor anteriorly and inferiorly, but did not penetrate the tumor (Fig. 1). Intraoperatively, encased vessels were also confirmed and resected. The tumor was totally resected with the attached falx.

Case 3: A 29-year-old woman presented with a 10year history of recurrent headache. MR imaging revealed heterogeneous enhancement of the tumor attached to the convex and falx in the right cerebral hemisphere. MR fusion imaging clearly showed that the $A_{2}$ and $A_{3}$ segments of the bilateral ACAs were displaced and ran along the posterior part of the tumor (Fig. 2). We confirmed and preserved the bilateral ACAs during surgery.

\section{Discussion}

This study showed that the new technique for fusing MR angiography and MR imaging allows clarification of the relationship between the intracranial vasculature and meningioma, which facilitates the sur-

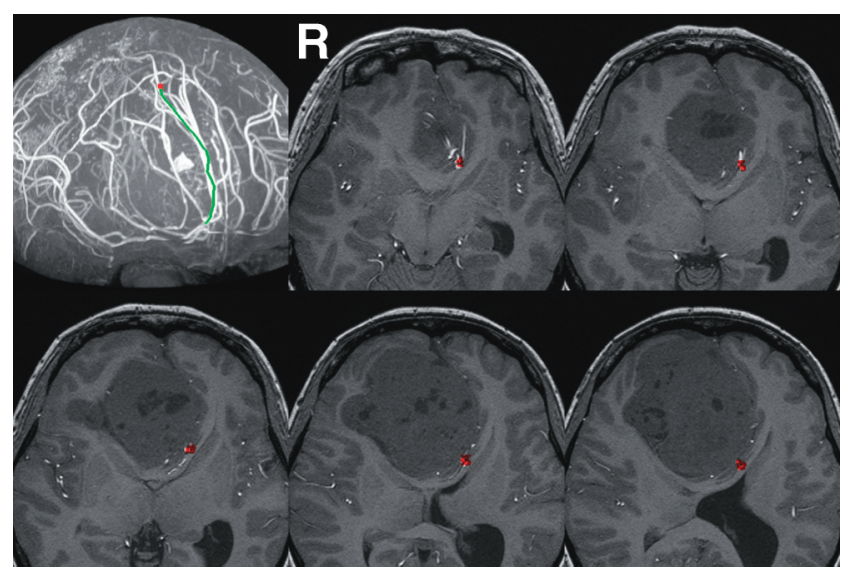

Fig. 2 Case 3, a 29-year-old woman with transitional meningioma. The green line was drawn on the anterior cerebral artery (ACA) on the maximum intensity projection image in three-dimensional space and the location of the green line was shown simultaneously on axial spoiled gradient recalled magnetic resonance images as a red point. The $A_{2}$ and $A_{3}$ segments of bilateral ACAs were displaced and ran along the posterior part of the tumor. 
gical planning.

Recently, image fusion techniques for registering different kinds of images have been developed in neuroimaging. ${ }^{4,8)}$ The interest in image fusion is based on the idea that creating a fused image combining multiple modalities may provide more information than analysis of these modalities separately. Previous studies have reported the combination of MR imaging with computed tomography (CT), intracranial echography, single photon emission CT, and positron emission tomography. ${ }^{4,8}$ Image fusion using 3D digital subtraction angiography (3D-DSA) and $3 \mathrm{D}-\mathrm{MR}$ imaging provides information about both intracranial soft tissues and vasculature, and can visualize any perforating artery visible on 3DDSA. Selecting any point on any of the 3D-DSA, and axial, coronal, and sagittal MR images can indicate the corresponding point on all the 3 other images. ${ }^{7)}$ 3D-DSA is the best technique to provide anatomical details of the vasculature, ${ }^{3,9)}$ but is invasive and involves a risk of neurological complications. ${ }^{1,10)}$

The present software fuses 2D-MR imaging with MR angiography to trace the vessels in 3D space and indicate the location of the vessel on the axial image simultaneously. This technique can easily demonstrate in real time which arteries on 3D-MR angiography correspond to hyperintense spots or strings on axial MR imaging, and vice versa. This technique is very useful for clarifying spatial relationships between the adjacent vasculature and lesions on axial MR imaging.

Fusion imaging as described here offers the advantage that different kinds of images can be obtained from the same modality. Registration procedures are thus readily performed. In contrast, the present technique possesses some limitations. While MR angiography can demonstrate arterial main trunks, the visibility of important smaller vessels such as the lenticulostriate arteries or anterior choroidal artery is inadequate. ${ }^{11)}$ Clarifying relationships between adjacent smaller vessels and lesions is thus difficult using this technique.

\section{Acknowledgments}

This work was supported in part by Grants-in-Aid for Advanced Medical Science Research by the Ministry of Education, Culture, Sports, Science, and Technology, Japan (2004-2008).

\section{References}

1) Cloft HJ, Joseph GJ, Dion JE: Risk of cerebral angiography in patients with subarachnoid hemor- rhage, cerebral aneurysm and arteriovenous malformation: a meta-analysis. Stroke 30: 317-320, 1999

2) Heiserman JE, Drayer BP, Keller PJ, Fram EK: Intracranial vascular stenosis and occlusion: evaluation with three-dimensional time-of-flight MR angiography. Radiology 185: 667-673, 1992

3) Hochmuth A, Spetzger U, Schumacher M: Comparison of three-dimensional rotational angiography with digital subtraction angiography in the assessment of ruptured cerebral aneurysms. AJNR Am J Neuroradiol 23: 1199-1205, 2002

4) Julow J, Major T, Emri M, Valálik I, Sági S, Mangel L, Németh G, Trón L, Várallyay G, Solymosi D, Hável J, Kiss T: The application of image fusion in stereotactic brachytherapy of brain tumours. Acta Neurochir (Wien) 142: 1253-1258, 2000

5) Kashimura $H$, Inoue $T$, Ogasawara $K$, Arai $H$, Otawara Y, Kanbara Y, Ogawa A: Prediction of meningioma consistency using fractional anisotropy value measured by magnetic resonance imaging. J Neurosurg 107: 784-787, 2007

6) Sekhar LN, Javed T: Meningiomas with vertebrobasilar artery encasement: review of 17 cases. Skull Base Surg 3: 91-106, 1993

7) Shimizu S, Suzuki H, Maki H, Maeda M, Miya F, Benali K, Trousset Y, Taki W: A novel image fusion visualizes the angioarchitecture of the perforating arteries in the brain. AJNR Am J Neuroradiol 24: 2011-2014, 2003

8) Stokking R, Zuiderveld KJ, Hulshoff Pol HE, van Rijk PP, Viergever MA: Normal fusion for three-dimensional integrated visualization of SPECT and magnetic resonance brain images. J Nucl Med 38: 624-629, 1997

9) Sugahara T, Korogi Y, Nakashima K, Hamatake S, Honda S, Takahashi M: Comparison of $2 \mathrm{D}$ and 3D digital subtraction angiography in evaluation of intracranial aneurysms. AJNR Am J Neuroradiol 23: 1545-1552, 2002

10) Willinsky RA, Taylor SM, TerBrugge K, Farb RI, Tomlinson G, Montanera W: Neurologic complications of cerebral angiography: prospective analysis of 2899 procedures and review of the literature. Radiology 227: 522-528, 2003

11) Wilms G, Bosmans H, Marchal G, Demaerel P, Goffin J, Plets C, Baert AL: Magnetic resonance angiography of supratentorial tumours: comparison with selective digital subtraction angiography. Neuroradiology 37: 42-47, 1995

12) Yano S, Kuratsu J; Kumamoto Brain Tumor Research Group: Indications for surgery in patients with asymptomatic meningiomas based on an extensive experience. J Neurosurg 105: 538-543, 2006

Address reprint requests to: Hiroshi Kashimura, M.D., Department of Neurosurgery, Iwate Medical University School of Medicine, 19-1 Uchimaru, Morioka, Iwate 020-8505, Japan.

e-mail: hkashi@iwate-med.ac.jp 


\section{Commentary}

The authors have to be congratulated for having fused the images of MR imaging, with the images of MRA. It is well known that the main problem for the parasagittal meningiomas, and in particular in the parietal region, are the bridging veins and how much they are in conflict with the tumorous lesion. It is, of course, very important also to be aware preoperatively of how the arteries are either incased in the tumor or pushed and stretched away.

This report does represent a very important practical step forward regarding more accurate planning of the surgical procedure in parasagittal meningiomas. In my personal view it is necessary to value the veins equally seriously as the arteries of the region.

Vinko V. DOlEnC, M.D. Department of Neurosurgery University Medical Center Ljubljana, Slovenia

The use of various fusion techniques has received considerable attention in the current literature and has started to yield encouraging results in clinical practice. Recent papers have described techniques for fusing 3D DSA images with MR images, PET with CT, PET with MRI and CT with MRI. Fusion techniques provide the information from two different radiological techniques in a single image.

In this paper, the authors describe a novel fusion technique for combining conventional MR imaging and MR angiography to facilitate surgical planning of meningiomas in 10 patients. Two illustrative cases have been discussed. The angiograms are obtained using the 3D TOF technique and fused with SPGR datasets to depict the relationship of the tumor with the intracranial vasculature. The authors have developed volume registration and visualization software to fuse the data on a personal computer.

The major limitation of this technique is inability to resolve smaller vessels and depict their relationship with the tumor. Thus, small vessels like the lenticulostriate vessels and the anterior choroid vessels and their relation to a tumor cannot be depicted. These small vessels can be depicted on a 3D DSA study, which can also be fused with conventional MR images. However, the highlight of this fusion software is that when a vessel is marked as a line on the $3 D \mathrm{MR}$ angiogram, it is depicted simultaneously on the axial SPGR image as a red point. This provides a unique anatomical perspective. Furthermore, the software developed by the authors supports images in the DICOM format in which they are acquired, thus limiting post processing times. A significant advantage of this technique is that the fusion imaging can be performed within 15 minutes. These fusion images can facilitate pre-treatment estimation of the functional risks related with vascular injuries by neurosurgical or endovascular treatment of any intracranial lesion.

Thus, fusion of conventional high resolution MR images with 3D MR angiograms described by the authors is an elegant and useful tool for the surgical mapping of the peritumoral angioarchitecture of a meningioma.

Darshana SANGHVI, M.D. and Atul Goel, M.D. Department of Neurosurgery King Edward VII Memorial Hospital \& Seth G.S. Medical College Parel, Mumbai, India

In contrast with the less complicated finding of vascular displacement, vascular invasion is one of the most important considerations in the preoperative evaluation to assess the resectability and risk of surgical treatment of complex intracranial tumors, especially meningiomas of the skull base. Kashimura et al. make an important contribution in their documentation that the fusion of magnetic resonance angiography and imaging studies applied preoperatively is of extraordinary value in predicting safe and effective resection of complex meningiomas. The authors report the technique is especially valuable for lesions that involve the proximal branches of the carotid, middle cerebral, and anterior cerebral arteries. At the Mayfield Clinic and the University of Cincinnati Neuroscience Institute, we have corroborated the authors' findings in several critical cases.

John M. TEW, Jr., M.D.

The Neuroscience Institute:

University of Cincinnati College of Medicine and the Mayfield Clinic Cincinnati, Ohio, U.S.A. 